http://bjas.journals.ekb.eg

\title{
Transfibular Approach for Ankle Arthrodesis
}

A.M.Halawa, E.S.Hussein, and M.M.A.Elshafey

Orthopedic Surgery, Dept., Faculty of Medicine, Benha Univ., Benha, Egypt. E-Mail:drma7moudmokhtar2013@gmail.com

\begin{abstract}
Background: Ankle arthritis is a highly frequent condition and treatment choice in young individuals may be a difficulty. Fifty percent of older people get some sort of foot or ankle arthrosis. The most prevalent cause is posttraumatic arthritis, comprising 70 percent of people with osteoarthritis. Ankle fusion gives a solid foot that is painless, plantigrade. Several various procedures for ankle fusion were documented, although, including external fixators, internal binding and intramedullary nailing, this is a rescue treatment that leads to lasting abnormalities in gait and there is a danger of worsening owing to the danger of developing ipsilateral hind foot arthritis. There are several operational procedures including open or arthroscopic methods for ankle arthrodesis. Following both techniques, successful clinical outcomes may be attained. The major purpose of this research was to assess the clinical impact of arthrodesis of the knee with transfibular fastening. Methods: Methods: This is a study of 20 patients operated on a transfibular basis at university hospitals Benha and El helal. Results and findings: Compared to the pre-operative values, the post-operative AOFAS rear foot score has been significantly improved. In 20 patients, $95 \%$ bone union was accomplished which demonstrates that ankle arthrodesis with transfibular fixation results in good clinical results. This method guarantees high bone union rate and strong stability, removes ankle discomfort, corrects deformation and enhances the ankle function substantially.
\end{abstract}

Keywords: Transfibular; Ankle; Arthrodesis.

\section{Introduction}

Ankle Arthrodesis is a conventional surgical procedure to treat posttraumatic ankle arthritis of rheumatoid arthritis (RA), infection, neuromuscular and failed complete arthroplasty of the ankles. Ankle fusion continues to be the preferred therapy for individuals with severe and continuous exercise and the replacement of ankles may only be advised for people with more moderate requirements[1].

Ankle fusion gives a solid foot that is painless, plantigrade. Several various procedures for ankle fusion have been documented; including external fixation, internal fixation, and intramedullary nailing, nevertheless, it is a recovery process that produces lasting changes in gait and the risk of ipsilateral reverse arthritis[2] may deteriorate in gait.

The therapy of ankle arthrosis includes alternatives for non-chirurgy and surgery. Nonsurgical therapy may include limiting and adjustment of activity, anti-inflammatory non-steroid medications, chondroprotective medicines such as glucosamine, cane or crutches, physiotherapy, shoe modification, orthosis and local injections[3].

There are several operational procedures including open or arthroscopic methods for ankle arthrodesis. Following both procedures, successful clinical results may be attained.

The approach to knee arthrode is separated among arthroscopic and open procedures. The open approach is further separated into the previous strategy, the post-competitive strategy, transfibular approach, media approach and combination lateral and medial approach. Compared to arthroscopy, the major advantage of an open method is that malalignment is less easily corrected and plate, screw and graft is easily applied. Open arthrodesis is linked with increased incidence of wound complications because of the large quantity of soft tissue dissection needed.
This may lead to extended hospitalisation and rehabilitation. [4]

Horwitz originally described the transfibular technique to ankle fusion. Adams reported transfibular arthrodesis via lateral approach, which removed the distal fibular fragment and prepared for use as a graft. The fibular graft offers good stability and scaffolding for Bone Bridge stimulation throughout the joint. In order to produce a suitable contact surface, the articular cartilage of the tibial ceiling and the talar dome is excised parallel to each other. In ankles where bone quality was low or bone defects occurred, graft from osteomised fibula was employed. Two cancellous $6,5 \mathrm{~mm}$ screws are utilised to neutrally compress the two surfaces of the arthrodesis. Two cortical $3.5 \mathrm{~mm}$ screws were utilised to compress the distal fibular onlay grease[5].

The objective of the investigation is to evaluate the outcomes using the transfibular method of ankle arthrodesis.

\section{Patients and methods}

This is a conducted study on twenty patients operated using transfibular approach at Benha university hospitals and El helal hospital.

The cases included in the study were:

- Advanced stage of ankle arthritis

- Avascular necrosis of talus

- Damage of articular cartilage after healed septic arthritis ankle

- Foot drop with tibialis posterior dysfunction

- Both genders are included

- Age :18 - 60

\subsection{The cases excluded from the study were}

- Arthritis of adjacent joints

- Active infection

- Peripheral vascular disease

- Peripheral neuropathy 


\subsection{Operative technique}

\section{Transfibular Approach and Exposure}

A longitudinal skin incision was made, starting 10 $\mathrm{cm}$ proximal to the tip of the lateral malleolus and continuing along the posterior border of the fibula in a distal direction. At the level of the tip of the lateral malleolus, the incisions continue in an anterior direction toward the base of the fourth metatarsus and ended at the level of the cuboid. Subperiosteal dissection was performed to expose the distal fibula to the lateral malleolus. Then the distal fibula was released from all attachments. At a distance of 6-7 cm

The lamina spreader was used to expand the operation field, the articular cartilage of tibia and talus was removed parallel to one another using an osteotome forming a smooth cancellous bone surface, and the 4.0-mm Kirschner wire was used to drill the tibia and talus.

\subsection{Fixation and Reconstruction}

After adequate preparation of the joint surfaces, the ankle joint was positioned sagittally in neutral, 0 to 5 degrees hindfoot valgus and external rotation equal to the opposite side if normal, or approximately 5 to 10 degrees if abnormal. Temporary fixation of the tibia and talus was accomplished with Kirschner wire from the tip of the lateral malleolus, saw was used to cut off and remove the distal end of the fibula. Then the tibiotalar joint was exposed with the help of a spreader. Next, the osteophytes were cleaned up and the articular cartilage was removed. The distal end of the fibula was split in the coronal plane, and the cancellous bone was removed completely and trimmed into cancellous bone particles, which were used for bone grafting of the tibiotalar joint if needed.

\subsection{Tibia and Talus preparation}

under fluoroscopy after achieving a satisfactory force line. Two $6.5 \mathrm{~mm}$ cancellous screws from anteromedial and anteolateral portions of tibia into the body of talus used to compress the two arthrodesis surface with the ankle in adequate position. The position was checked with the image intensifier intraoperatively (Anteroposterior and lateral views). In ankles in which bone quality was poor or there were bone defects, graft was used from the osteomtized fibula.Then the fibula was fixed as onlay graft to the tibia and talus with two $3.5 \mathrm{~mm}$ cortical screws. After the surgery, the surgical incision was closed using the conventional method, and a below knee back-slab was applied.
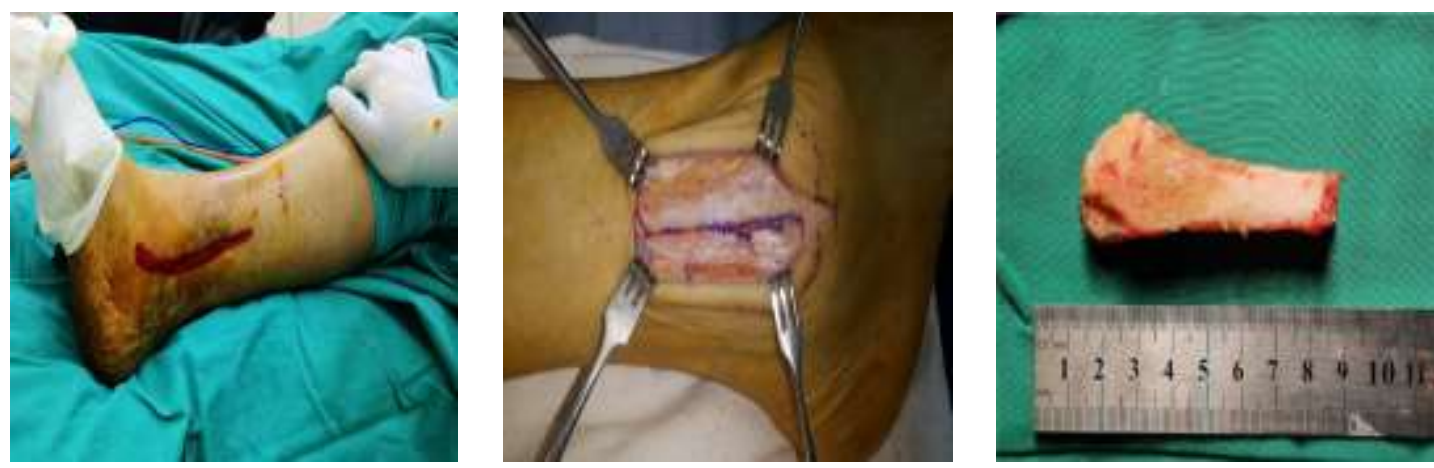

Fig. (1) Intraoperative photographs showing Transfibular Approach and Exposure.

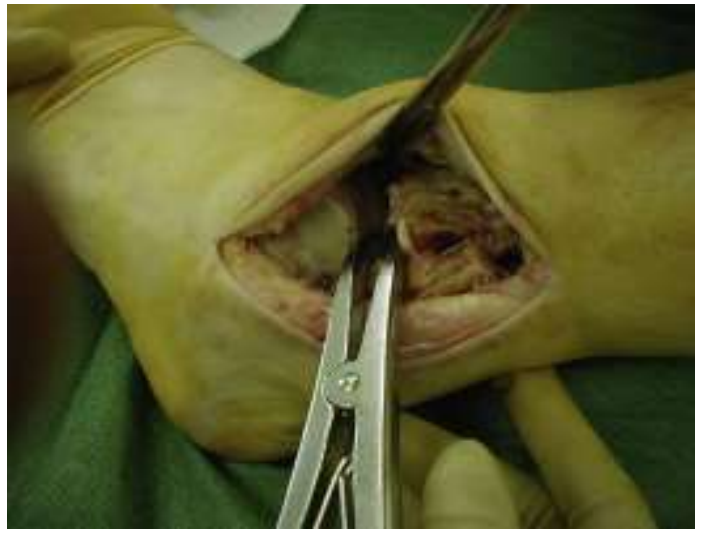

Fig. (1) Intraoperative photographs showing exposure of the ankle joint by lamina spreader. 


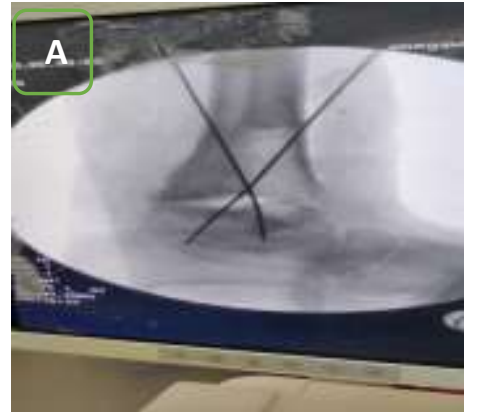

A: Intraoperative photo on the image intensifier showing configuration of guide wires on AP view.

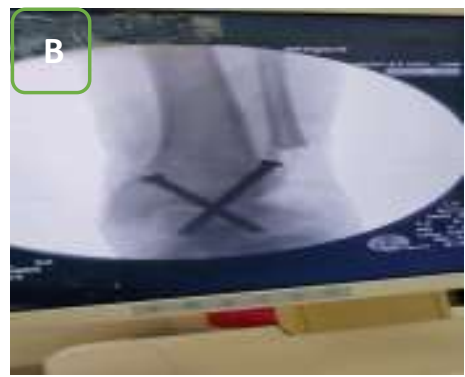

B: Intraoperative photo on the image intensifier showing configuration of the two cannulated screws on AP view.

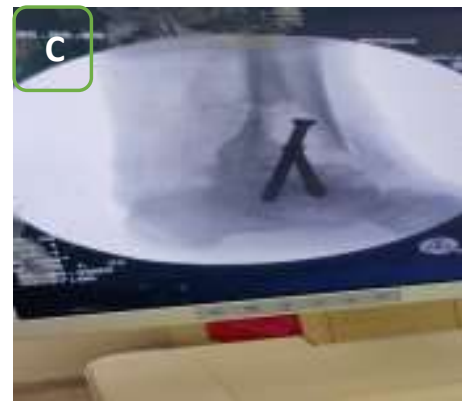

C: Intraoperative photo on the image intensifier showing configuration of the two cannulated screws on lateral view.

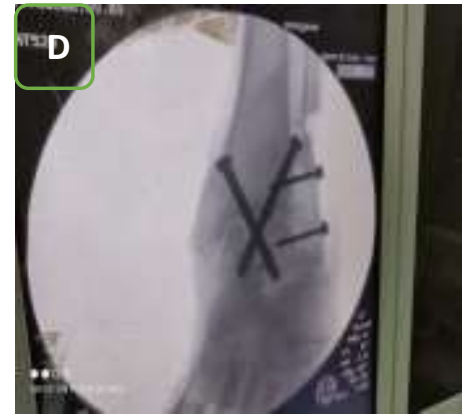

D: Intraoperative photo on the image intensifier showing after fixation of the fibule as onlay graft with cortical screws on AP view.

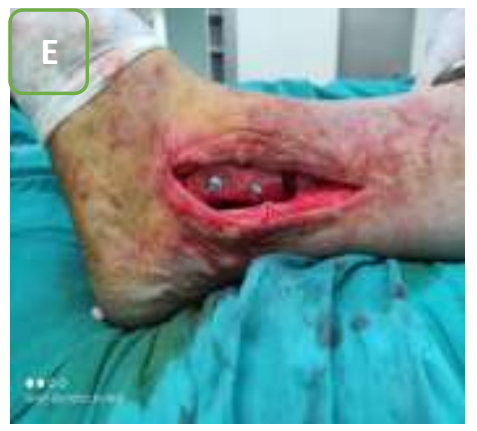

E: Intraoperative clinical photo showing after fixation of the fibule as onlay graft with cortical screws.

Fig. (3) showing Fixation and Reconstruction. 


\subsection{Postoperative care}

All patients were given one daily dose of lowmolecular weight heparin. All the patients had postoperative oral antibiotics for 5 days, together with analgesic and anti-edematous drugs. The foot was protected by below knee slab. Sutures were removed after 2 weeks. They were kept non-weight bearing for 6 weeks in below knee cast. Then a removable boot was applied and the patient was allowed partial weight bearing for 6 weeks. At 12 weeks after surgery if clinical and radiological signs of fusion were present the patient could return to daily activities.

\subsection{Post-operative radiological evaluation}

Follow up x-ray is done at 3 weeks, 6 weeks, 12 weeks and 6 months.

\section{Postoperative clinical follow up}

Clinical evaluation was standardized for all patients at 3 months and 6 months postoperatively. The patients were evaluated by American orthopedic foot and ankle society (AOFAS) hind foot score. Postoperative evaluation also includes the postoperative hospital stay, complications, time of weight bearing and the need for further hospital admission.

\subsection{Statistical analysis}

Gathered data will be processed using SPSS version 21 (SPSS Inc., Chicago, IL, USA). Quantitative data will be expressed as means \pm SD while qualitative data will be expressed as numbers and percentages (\%). Student $t$ test will be used to test significance of difference for quantitative variables and Chi Square will be used as a test of significance of difference for qualitative variables. A probability value $(p-v a l u e)<0.05$ will be considered statistically significant.

\section{Results}

The mean age was 46.8 years ranging from 35 to 60 years and according to gender distribution, they were $13(65.00 \%)$ males and $7(35.00 \%)$ females. The mean BMI was $24.8 \pm 0.7$. $60 \%$ of patients were Smokers. Table (1)

The mean preoperative pain score was 5.5 (ranging from 0 to 40 ). The mean pain score at final follow up was 36.0(ranging from 20 to 40). There was significant improvement in pain score in the final follow up as compared to pre- operative pain score ( $p$ value $<0.001$ ). As regard pain at final follow up 14 patients had no pain, 4 patients had mild pain and only 2 patients had moderate pain table (2).

The mean preoperative activity score was 3.3 (ranging from 0 to 7 ). The mean activity score at final follow up was 7.4 (ranging from 4 to 10). There was significant improvement in activity score in the final follow up as compared to pre- operative activity score ( $\mathrm{p}$ value $<0.001$ ). At final follow up there were no limitation of daily activity in 17 patients. However, limited daily activity was observed in 3 . table (3).

Table (1) Demographic data among the patients.

\begin{tabular}{llcc}
\hline & Mean \pm SD & \multicolumn{2}{c}{$46.8 \pm 15.6$} \\
Age (years) & Range & 13 & $35-60$ \\
Gender & Male & 7 & $65 \%$ \\
BMI & Female & 12 & $24.8 \pm 0.7$ \\
Smoking & & 12 & $60 \%$ \\
\hline
\end{tabular}

Table (2) Showing preoperative and final follow up score for pain.

\begin{tabular}{llccc}
\hline & & Pre-operative & final follow up & P Value \\
\cline { 2 - 5 } & Severe (0) & $17(85 \%)$ & 0 & \\
& Moderate (20) & 0 & $2(10 \%)$ & \\
Pain & Mild (30) & $1(5 \%)$ & $4(20 \%)$ & $<0.001$ \\
& None (40) & $2(10 \%)$ & $14(70 \%)$ & \\
\hline
\end{tabular}

Table (3) Representing activity limitation preoperative and at final follow up of the patients involved in the study.

\begin{tabular}{|c|c|c|c|}
\hline & Pre-operative & final follow up & P Value \\
\hline Severe limitation of daily activit(0) & $5(25 \%)$ & 0 & \\
\hline some limitation of daily activity (4) & $13(65 \%)$ & $3(15 \%)$ & \\
\hline Recreational activity limitation (7) & $2(10 \%)$ & $12(60 \%)$ & \\
\hline No limitation (10) & 0 & $5(25 \%)$ & $<0001$ \\
\hline Mean \pm SD & $3.3 \pm 2.112$ & $7.4 \pm 1.74$ & $<0.001$ \\
\hline
\end{tabular}


The mean preoperative score for maximum walking distance was 2.3 (ranging from 0 to 4 ). The mean score for max walking distance at the final follow up was 4, 45 (ranging from 2 to 5). There was significant improvement at the final follow up max walking distance as compared to pre-operative score ( $p$ value $<0.001$ ). At the final follow up 11 patients could walk unlimited distance, 8 patients could walk less than 6 blocks and 1 patient could walk less than 4 blocks table (4)

The mean preoperative score for assessment of gait abnormalities was 1.6 (ranging from 0 to 4 ). The mean score at the final follow up was 6.8 (ranging from 4 to 8 ). There was significant difference ( $\mathrm{p}$ value $<0.001$ ). Slight gait abnormalities were noted in 14 patients and 6 patients show obvious abnormalities in the gait table (5)

The mean preoperative total score was 31.35 (ranging from 17 to 65 ). The mean total score at final follow up was 82.15 (ranging from 44 to 92). There was significant difference (p value < 0.001) Fig.(4).

Clinical signs of union were taken to be absence of pain and no movement at the ankle on stressing it in different planes. 19 patients $(95 \%)$ showed clinical signs of union with average time of clinical union was at 10 weeks and ranged from 8 to 14 weeks. Radiological signs of union were noted in 19 patients $(95 \%)$ at the final follow up after 6 months with average time of union 11 weeks and ranged from 8 to 14 weeks. At 8 weeks $50 \%$ of patients show clinical and radiological signs of union, at 10 weeks $70 \%$ of patients show clinical and radiological signs of union, at 12 weeks $90 \%$ of patients show clinical and radiological signs of union and at 14 weeks $95 \%$ of patients show clinical and radiological signs of union.

Nonunion occur in $5 \%$ of cases (1 patient) which assessed both clinically and radiologically.

Superficial wound infection occurred in 2 patients $(10 \%)$. Infection was discovered in the first follow up visit at second week after surgery. Infection was controlled after intensive course of antibiotics for 1 month begin first parenteral for one week and continued on oral for another 3 weeks until infection completely subsided.There were three patients with hardware complication as the tip of guide of cannulated screws broke intra-operatively. It was not removed as it was completely inside the calcaneus and did not cause any problem. Adjacent joint arthrosis and deep venous thrombosis did not occur in any cases. table (6)

Table (4) Representing max walking distance preoperative and at final follow up of the patients involved in the study.

\begin{tabular}{lccc}
\hline & Pre-operative & final follow up & P Value \\
\hline$<$ 1 block (0) & $2(10 \%)$ & 0 & \\
$\mathbf{1 - 4}$ blocks (2) & $13(65 \%)$ & $1(5 \%)$ & \\
4-6 blocks (4) & $5(25 \%)$ & $8(40 \%)$ & \\
$>$ 6 blocks (5) & 0 & $11(55 \%)$ & $<0.001$ \\
Mean \pm SD & $2.3 \pm 1.557$ & $4.45 \pm 0.83$ & \\
\hline
\end{tabular}

Table (5) Representing gait abnormality preoperative and at final follow up of the patients involved in the study.

\begin{tabular}{lccc}
\hline & Pre-operative & final follow up & P Value \\
\hline Marked (0) & $12(60 \%)$ & 0 & \\
Obvious (4) & $8(40 \%)$ & $6(30 \%)$ & $<0.001$ \\
None (8) & 0 & $14(70 \%)$ & \\
Mean \pm SD & $1.6 \pm 2.02$ & $6.8 \pm 1.84$ & \\
\hline
\end{tabular}

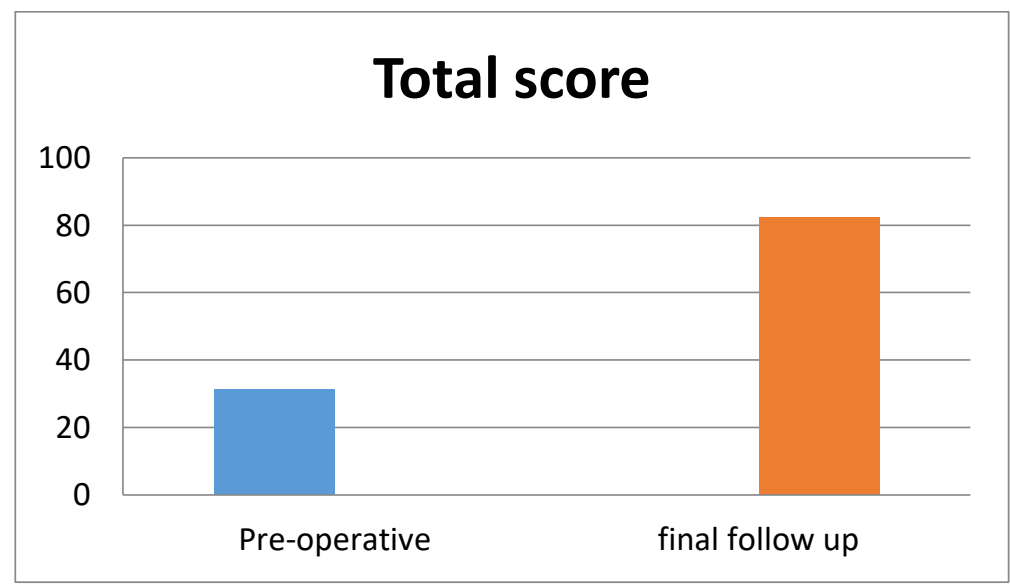

Fig. (2) Graph representing total score preoperative and at final follow up. 
Table (6) Complications which occurred in the patients involved in our study.

\begin{tabular}{ll}
\hline Complications & No of patients \\
\hline Non union & 1 \\
Superficial wound infection & 2 \\
Hardware complications & 3 \\
Adjacent joint arthrosis & 0 \\
Deep venous thrombosis & 0 \\
\hline
\end{tabular}

\section{Discussion}

Our investigation was conducted in an intervention trial in 20 participants with ankle arthritis (18) and foot drop (2). The age ranged from 35 to 60 years and, based on the distribution of sex, was 46.8 years for $13(65 \%)$ men and $7(35 \%)$ females. The average $\mathrm{BMI}$ is $24.8 \pm 0.7$.

A total AOFAS score was done for the clinical assessment of patients pre-operatively and postoperatively. Then we compare the outcomes among them.

The average overall AOFAS value for our research was 31,35 . (ranging from 17 to 65). The average final score was 82,15 . (ranging from 44 to 92).

Haiqiang Suo et al[6] had a mean total AOFAS preoperative score of $43,46 \pm 4,39$. The average final score was $80,39 \pm 5,37$.

Dong yeon lee et al.[7], the average total AOFAS preoperative value was 58,80 . (22-89). In the end, the average total score was 79, 83. (53-100).

The average preoperative AOFAS total score for Kim et al.[8] was 44, 5 (32-60). The average score at the end was 60,7 (48-86).

Balaji et al[9] have not provided the average overall preoperative AOFAS score. At the last followup, the average score was 74 .

Lee et al [10], the mean total AOFAS preoperative score was 30 . $(0-47)$. The average final follow-up score was 71 (38-87).

The clinical symptoms of union were taken as the absence of discomfort and no ankle mobility in various planes to stress it. There have also been radiological symptoms of union in patients.

Our union rate of research was 95 percent, compared to Haiqiang Suo et al[6], with a union rate of 100 percent. It was similarly equivalent to Dong Yeon Lee et al [7] with a union rate of 100 percent. It was equivalent to Kim et al [8] with a union rate of 95 percent. It was equivalent to Balaji et al[9] with a union rate of $100 \%$. It was likewise similar to Lee et al[10] with a union rate of 92 percent.

Nonunion occurs in $5 \%$ of cases (1 patient) with clinical and radiographic evaluation. In 2 individuals, superficial wound infection occurred (10 percent ). The infection was identified in the second week after surgery at the initial follow-up appointment. After 1 month of vigorous antibiotic course, the infection was managed for one week at first parenteral and maintained for another 3 weeks orally till infection fully eliminated. The guiding tip for cannulated screws was broken intra-operatively by three patients with hardware complications. It was not entirely removed since it was within the calcaneus and caused no problems.

Haiqiang Suo et al[6] had a superficial peroneal nerve paralysis following surgery, which was mostly exhibited as numbness and discomfort on the lateral calf and rear of the foot, and a substantial loss in feeling when touched. This problem has been resolved 1 year after surgery with neurotrophic medicines. Healing of the skin of the incision was retarded in another patient who resolved with constant change of dressing within 1 month after the procedure.

Kim et al [8], Non-union occurs without problems in 1 case.

Lee et al [10], non-union occurs without problems in 1 patient.

In no instance did adjacent joint arthrosis develop in our investigation, however Lee et al [10]. One single patient Of the 23 instances, subtalar arthrodesis occurred.

The merits of this research include the use of the same pre-operative assessment for all patients. All patients were transfibularly operated.

The short follow-up time is a drawback of the current research (one year). This restricts the capacity to identify long-term difficulties caused by the neighbouring joint deterioration (subtalar, talonavicular, tarsometatarsal). The tiny sample size of this research is also limited. This may be improved in the future through multi-center studies.

\section{Conclusion}

The major purpose of this research was to assess the clinical impact of arthrodesis of the knee with transfibular fastening. Compared to the preoperative values, the post-operative AOFAS rear foot score has been significantly improved. In 20 patients, $95 \%$ bone union was accomplished which demonstrates that ankle arthrodesis with transfibular fixation results in good clinical results. This method guarantees high bone union rate and strong stability, removes ankle discomfort, corrects deformation and enhances the ankle function substantially.

\section{References}

[1] A.Haskell, "Total Ankle Arthroplasty: The US Experience," in International Advances in Foot and Ankle Surgery, Springer, vol. 2012, pp. 467487, 2012. 
[2] K.M.Dupont, N.Shibuya, and J.T.Bariteau, "Tibiotalocalcaneal arthrodesis with intramedullary nails-mechanobiological background and evolution of compressive technology," Glob J Ortho Res, vol. 1, pp. 873 884, 2019.

[3] M.H.Gilzad-Kohan and F. Jamali, "Glucosamine and adjuvant arthritis: a pharmacokinetic and pharmacodynamic study," Eur. J. Pharm. Sci., vol. 47, pp. 387-393, 2012.

[4] T.R.Daniels . "Intermediate-term results of total ankle replacement and ankle arthrodesis: a COFAS multicenter study," JBJS, vol. 96, pp. 135-142, 2014

[5] Y.Sripanich, J.Steadman, V.Valderrabano, and A. Barg, "Open Ankle Arthrodesis: Transfibular Approach," Tech. Foot Ankle Surg., vol. 19, pp. 26-36, 2020.

[6] H.Suo, L.Fu, H.Liang, Z.Wang, J.Men, and W.Feng, "End-stage Ankle Arthritis Treated by Ankle Arthrodesis with Screw Fixation Through the Transfibular Approach: A Retrospective
Analysis," Orthop. Surg., vol. 12, pp. 1108-1119, 2020.

[7] D.Y.Lee, M.G.Kyung, Y.J.Cho, S. Hwang, H. W. Kang, and D.-O. Lee, "A modified transfibular technique of ankle arthrodesis using partial fibular resection and onlay bone graft," PLoS One, vol. 15, p. e0241141, 2020.

[8] J.-G.Kim ."Ankle arthrodesis: a comparison of anterior approach and transfibular approach," Clin. Orthop. Surg., vol. 10, p. 368, 2018.

[9] S. M. Balaji, V. Selvaraj, S. Devadoss, and A. Devadoss, "Transfbular ankle arthrodesis: A novel method for ankle fusion-A short term retrospective study," Indian J. Orthop., vol. 51, pp. 75-80, 2017.

[10] H.-J. Lee, W.-K. Min, J.-S. Kim, S.-D. Yoon, and D.-H. Kim, "Transfibular ankle arthrodesis using burring, curettage, multiple drilling, and fixation with two retrograde screws through a single lateral incision," J. Orthop. Surg., vol. 24, pp. 101-105, 2016. 